\title{
VERIFICATION OF THE LINEAR MATCHING METHOD FOR LIMIT AND SHAKEDOWN ANALYSIS BY COMPARISON WITH EXPERIMENTS
}

\author{
James Ure \\ University of Strathclyde \\ Glasgow, UK, G1 1XJ \\ james.ure@strath.ac.uk
}

\author{
Haofeng Chen ${ }^{1}$ \\ University of Strathclyde \\ Glasgow, UK, G1 1XJ \\ haofeng.chen@strath.ac.uk
}

\author{
David Tipping \\ EDF Energy, Barnwood, \\ Gloucester, UK, GL4 3RS \\ david.tipping@edf-energy.com
}

\section{ABSTRACT}

The Linear Matching Method (LMM), a direct numerical method for determining shakedown and ratchet limits of components, has seen significant development in recent years. Previous verifications of these developments against cyclic nonlinear finite element analysis have shown favourable results, and now this verification process is being extended to include comparisons with experimental results.

This paper presents a comparison of LMM analysis with experimental tests for limit loads and shakedown limits available in the literature. The limit load and shakedown limits were determined for pipe intersections and nozzle-sphere intersections respectively, thus testing the accuracy of the LMM when analysing real plant components. Details of the component geometries, materials and test procedures used in the experiments are given. Following this a description of the LMM analysis is given which includes a description of how these features have been interpreted for numerical analysis. A comparison of the results shows that the LMM is capable of predicting accurate yet conservative limit loads and shakedown limits.

\author{
NOMENCLATURE \\ E Elastic Modulus \\ $\sigma \quad$ Stress \\ $\sigma_{\mathrm{y}} \quad$ Yield Stress \\ $\varepsilon \quad$ Strain \\ FEA Finite Element Analysis \\ LMM Linear Matching Method \\ Current Increment \\ i+1 Subsequent Increment
}

\section{INTRODUCTION}

Demonstration of shakedown is an integral part of the design and assessment of pressurised components. Several options exist to demonstrate this including the simplified routes based on elastic analyses, such as that in [1], and full cyclic non-linear Finite Element Analysis (FEA). The development of Direct Methods, based on the shakedown bounding theorems [2,3], has provided a third option to demonstrate shakedown. Included among these Direct Methods is the Linear Matching Method (LMM) [4$6]$.

These Direct Methods are becoming an increasingly popular method of demonstrating shakedown. There are many cases where the conventional simplified approach gives results which are overly conservative. The use of full non-linear FEA is not only computationally expensive but can also give ambiguous results in terms of the shakedown status of the component. The LMM provides solutions to these problems. Firstly, in the case of conservatism of results, both lower and upper bounds to the exact shakedown limit are provided and consistently converge to within a very small tolerance of each other [7]. The LMM also provides these solutions with less computational expense than a full non-linear solution, and the bounding theorem foundations mean that the basis for this solution is more concrete than the judgement and estimations often required with the full non-linear option. For these reasons, the LMM has been incorporated into the R5 high temperature research program of EDF Energy, with a view to including it within the R5 structural integrity assessment procedure [1].

\footnotetext{
${ }^{1}$ Corresponding author
} 
The LMM has been validated against full non-linear FEA on many occasions, and favourable results have been seen with all of these comparisons (in [7] for example). These comparisons are needed to verify that the method has been implemented correctly within the finite element framework. However, it is also important to verify the entire analysis tool. This includes assumptions and simplifications which are external to the FEA implementation, such as the use of simplified material models. To achieve this, comparisons against experiments are required.

This paper presents the comparison of experimentally determined limit loads [8] and shakedown limits [9] with those predicted by the LMM. The experiments were performed on pipe intersections and nozzlesphere intersections respectively, thus reproducing realistic geometries used in plant systems. After giving a brief introduction to the LMM, this paper describes limit load and shakedown limit tests alongside the strategy used to analyse these using the LMM. A comparison of the experimentally and numerically derived limits is given which demonstrates the ability of the LMM to predict accurate yet conservative limit loads and shakedown limits.

\section{THE LINEAR MATCHING METHOD}

The Linear Matching Method (LMM) has been fully described in other publications [4-6], and so only a brief outline of the shakedown method, used in this work, is given.

The basic premise of the LMM is that a nonlinear material response can be mimicked by a series of iterative elastic solutions where the modulus is modified within the volume of the structure to bring the stresses equal to the yield stress. Figure 1 demonstrates this pictorially.
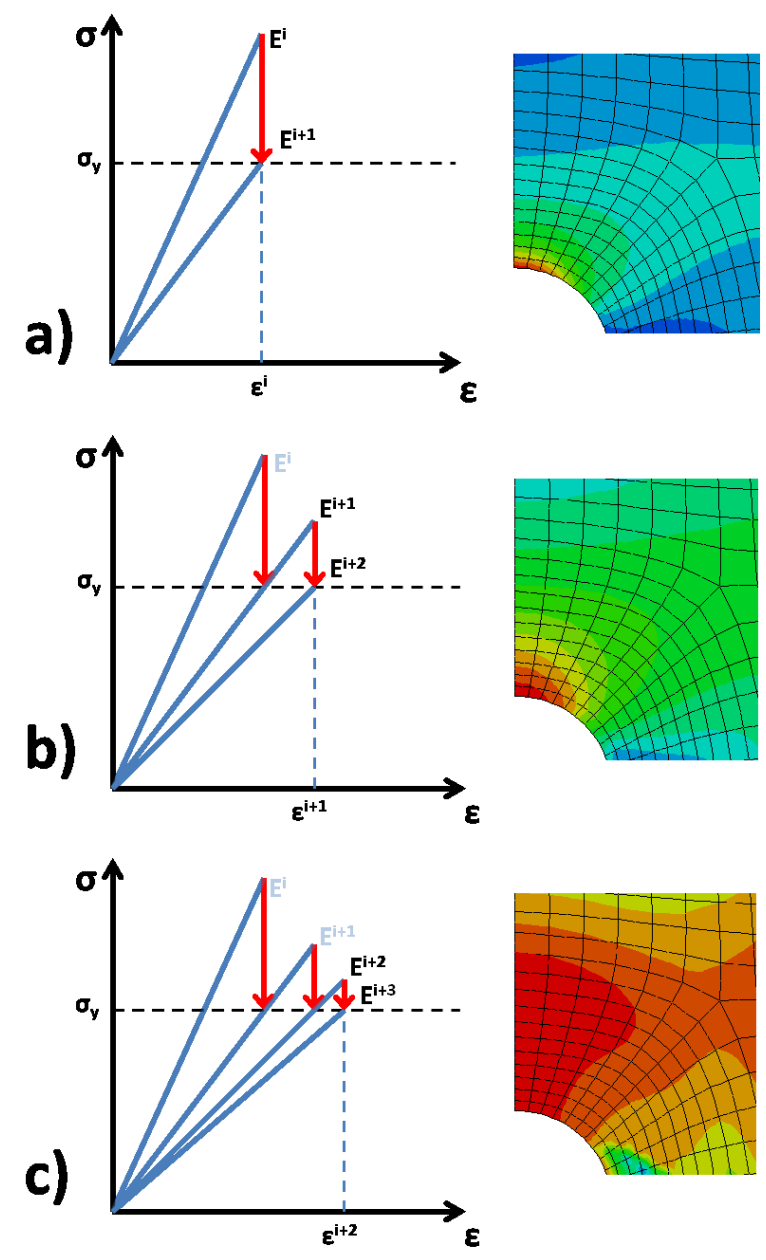

Figure 1 - Iterative Modulus Adjustment Procedure 
Figure 1a shows the initial elastic analysis for the applied loads. At each integration point the modulus is modified at a fixed level of strain such that the stress equals the yield stress. This updated modulus is then used in the second iteration (Figure 1b), where the modulus is once again adjusted to bring the stress to the yield stress. A very similar action is taken at points where the stress is below yield where the modulus is increased so that the stress is equal to yield. Repetition of this process allows the stresses to re-distribute in the structure in a very similar way to that of a non-linear material and also allows a constant residual stress field to form.

In conjunction with the modulus adjustment procedure the applied loads are scaled using an upper bound load multiplier, which is calculated based on Koiters Theorem [2]. The combined effect of the modulus adjustment and load scaling allows the LMM to converge towards the exact shakedown limit. Lower bounds to the shakedown limit are calculated by using Melan's Theorem [3] which ensures that the stresses from the applied loads satisfy the yield stress at all points in the model and at all points in the load cycle.

\section{LIMIT LOAD COMPARISONS}

The limit load tests used for comparison in this work are those performed by for the Welding Research Council, specifically the tests reported in WRC Bulletin 219 [8]. The limit loads of pipe intersections subject to internal pressure and in-plane bending moments were determined. A brief description of the manufacture and testing of the pipe intersections is given before a description of the LMM calculations and a results comparison.

\subsection{Experimental Tests}

The pipe intersections were machined from a single billet of hot-rolled steel plate of ASTM A-36 grade steel. The machining contained three steps: A rough cut to approximate dimensions, an anneal to remove the residual stresses both inherent in the parent plate and caused by machining, and a final finishing cut to bring the component to the final dimensions.

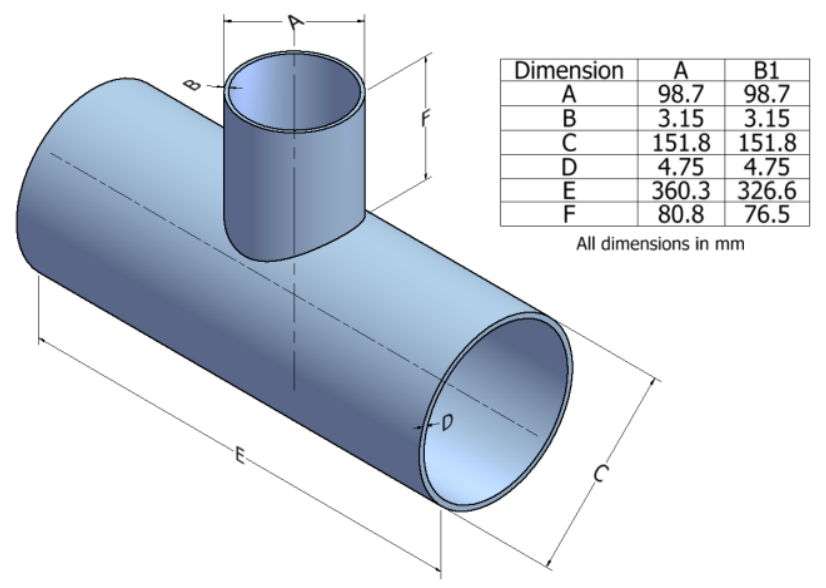

Figure 2 - Pipe Intersection Schematic and Dimensions

For continuity the naming of the intersections used in [8] will also be used here, and Figure 2 shows the final dimensions of the two intersections, A and B1, as quoted in [8].

The internal pressure testing of intersection A was achieved by welding plates to the open ends of the pipes and then using a pressure fitting in one of these ends to supply pressurised fluid. The moment loading of intersection B1 was applied to the intersecting pipe by two hydraulic rams acting in opposite directions. These, in turn, were connected via pin joints to a loading arm which was fixed to the free end of the intersecting pipe (as shown schematically in Figure 3a). This arrangement applied a pure moment couple to the pipe. Multiple dial gauges and strain gauges were fitted to the intersections for testing and were used to define the limit load of the components, see section 3.3. 


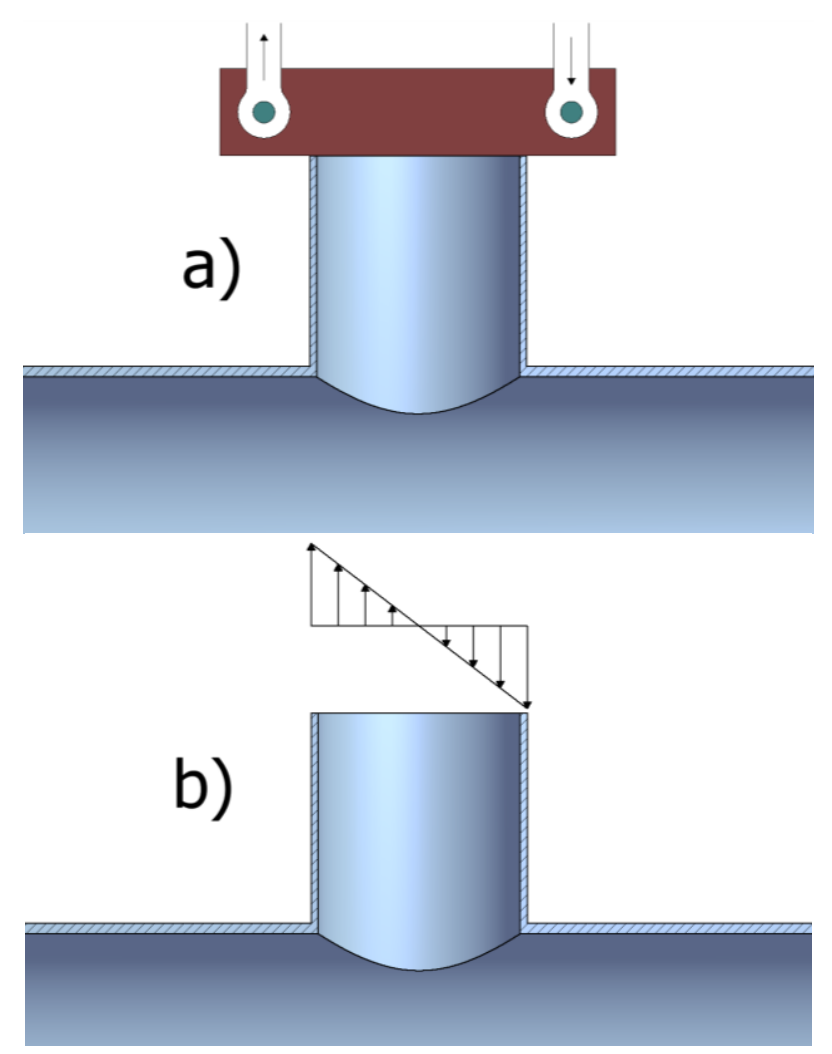

Figure 3 - Nozzle-Sphere Moment Application Schematic in a) Experiment and b) LMM Analysis

The material properties of the steel were obtained by machining tensile and compressive test specimens from the same billet as the intersections were manufactured from. (i.e. an individual yield stress was determined for each nozzle). These specimens were also subject to the same annealing treatment. Figure 4 shows a typical stress-strain response of the material tests and Table 1 shows the yield stresses $(0.2 \%$ offset strain) reported for each intersection. The reported yield stresses are the average of six tests, where a deviation of no more than $2 \%$ from the mean was observed in any test.

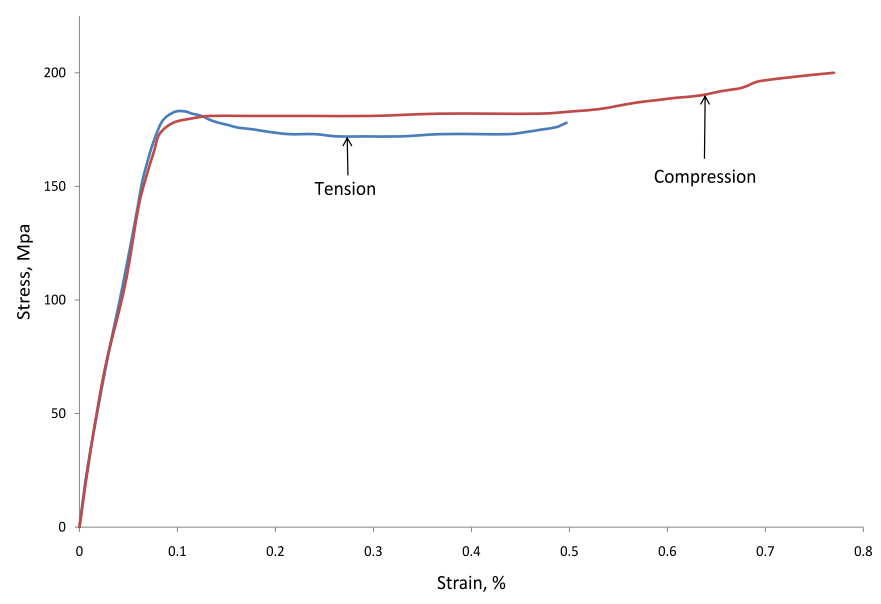

Figure 4 - Typical Stress-Strain Response of Intersection Material as reported in [8]

\subsection{Assessment Using the LMM}

Using the information given in [8], the pipe intersections were modelled in Abaqus [10] for assessment with the LMM. The dimensions of both intersections is reported with great accuracy, which allowed an 
exact geometry to be created and meshed in Abaqus CAE using symmetry where appropriate. Model A was modelled using a one-quarter model due to the uniform loading. Model B1 used one-half symmetry due to the symmetry of the applied moment loading along the axis of the main pipe (shown in Figure 5).

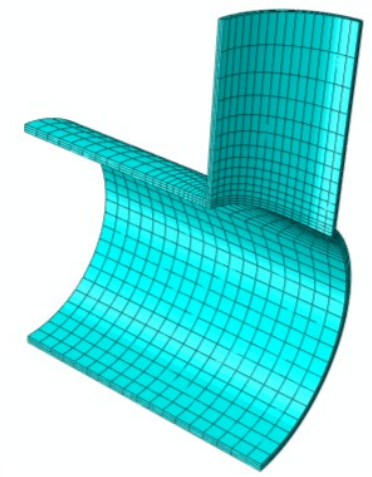

a)

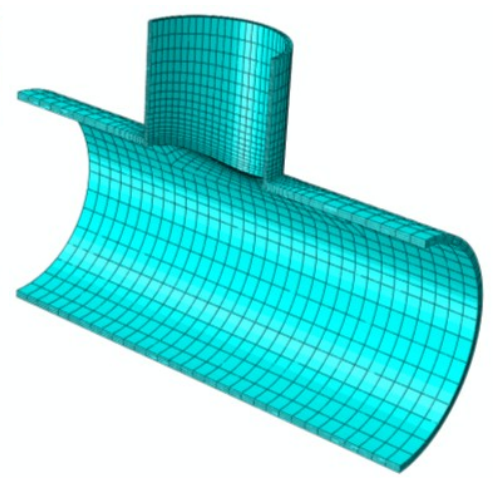

b)

Figure 5 - Nozzle-Sphere FEA Models for a) Model A and b) Model B1

A perfectly plastic material model was used for both limit pressure and limit moment analyses using the yield stress quoted in Table 1. Looking at Figure 4 it can be seen that for the range of strains shown in the tensile and compressive tests that a perfectly plastic material, despite being a very simple model, is a reasonable approximation to this material response.

Table 1 - Yield Stresses of Intersections A and B1
\begin{tabular}{|c|c|}
\hline Test & Yield Stress (MPa) \\
\hline A & 198 \\
\hline B1 & 167 \\
\hline
\end{tabular}

The loading and boundary conditions were chosen to most accurately represent the conditions of each test. In model A internal pressure loading was applied to all internal surfaces, and due to the closure of the ends in the tests, the closed end condition was applied in the model. To achieve this the equivalent axial tension was applied to the free ends of the pipes. Free radial expansion of the pipes was allowed, as per the tests, and the free ends of the pipes were constrained to remain in-plane during longitudinal expansion. In model B1, the ends of the main pipe were fully fixed. The bending moment was applied to the intersecting pipe using the DLOAD subroutine [10], which allowed a pure couple to be applied in the form of a linear pressure distribution across the free end, as shown in Figure $3 b$.

\subsection{Results Comparison}

In [8], values of limit load are quoted for each test, each of which is determined using a different interpretation of the load-strain and load-deflection data obtained during the test. In this work, the lowest of the three reported values is used for comparison to maintain a level of conservatism.

The LMM produces lower and upper bounds to the limit load, and both values are included here to demonstrate the level of convergence possible between the two values. In addition to the LMM values, the limit load calculated in a conventional Abaqus limit analysis (using the same mesh as the LMM analysis) is presented as an additional comparison. All of these results are shown in Table 2.

Table 2 - Limit Load Comparison

\begin{tabular}{|c|c|c|c|c|}
\hline & & \multicolumn{2}{|c|}{ LMM } & \\
\hline Test & $\begin{array}{c}\text { Abaqus } \\
\text { Limit }\end{array}$ & $\begin{array}{c}\text { Upper } \\
\text { Bound }\end{array}$ & $\begin{array}{c}\text { Lower } \\
\text { Bound }\end{array}$ & Experiment \\
\hline A & 7.19 & 7.19 & 7.12 & 8.0 \\
\hline B1 & 2970 & 2968 & 2939 & 3184 \\
\hline
\end{tabular}


Comparison of the limit load values in Table 2 reveals that a slightly conservative limit pressure and moment is predicted which also compares favourably to that predicted by the Abaqus limit analysis using the Riks method. Two conclusions can be drawn from these results. The first is that the implementation of the lower and upper bounding theorems in the LMM is correct, given that the LMM and Abaqus limit results match almost exactly. These three FEA results are all based on a different theoretical foundation, and so the uniformity of the results gives confidence in their individual ability to predict limit loads. This gives confidence that the LMM can be used in situations which cannot be directly compared to conventional FEA (assessment of the shakedown boundary for example) because the implementation of the bounding theorems has been verified. The second conclusion is that the LMM performs as well as conventional non-linear FEA methods for predicting limit loads with the same modeling approximations, such as simplified material models.

\section{SHAKEDOWN LIMIT COMPARISONS}

The ability to predict limit loads is a useful first verification as it serves to validate the implementation of the bounding theorems used in the LMM. The capabilities of LMM, however, extend beyond those of conventional FEA to the calculation of the shakedown limit, and so this functionality of the method must also be verified. To achieve this, the shakedown tests performed by the C.E.G.B. [9] are used.

\subsection{Experimental Tests}

The experiments performed in [9] investigated the shakedown pressure of nozzles in spherical shells. Two of these tests are discussed here, both of which made use of oblique nozzles in the shell. Once again the naming convention in the original report has been adopted in order to maintain consistency, and Nozzles 5 and 6 are considered in this work.

Table 3 - Material Properties of Nozzle and Shell Materials

\begin{tabular}{|c|c|c|}
\hline Material & $\begin{array}{c}\text { Yield Stress } \\
(\mathrm{MPa})\end{array}$ & $\begin{array}{c}\text { Ultimate Tensile } \\
\text { Strength (MPa) }\end{array}$ \\
\hline Nozzle & 265 & 493 \\
\hline Shell & 273 & 485 \\
\hline
\end{tabular}

The vessels were manufactured from boiler plate (shell material) and forged bar (nozzle material) which were chosen to have closely matched mechanical properties. The yield stresses and ultimate tensile stresses of the two materials given in [9] are shown in Table 3 and Figure 6 shows the dimensions of the two geometries.

The tests were performed to find the shakedown pressure of these nozzles. Many strain gauges were attached to the nozzles prior to testing and these strain readings were used to determine the shakedown status of the vessel.

Beginning at ambient pressure, the vessel was pressurized to the current test pressure and then back to ambient conditions. The initial pressure cycle began at ambient, pressurized to 400psi and then returned to ambient. If shakedown was observed with this level of pressure cycling, then the maximum pressure in the cycle was increased by 50psi and the cycling was repeated. In these tests shakedown was said to occur when identical strains were recorded in three consecutive cycles. If this shakedown criterion was not met within 8 pressure cycles, it was concluded that the vessel would not attain shakedown.

\subsection{Assessment Using the LMM}

The geometry of the nozzles was modeled in Abaqus CAE where the dimensions of the welds (not fully documented in the published results) were estimated based on likely leg lengths for the thickness of the shell and nozzle. The symmetry of both nozzles is used by creating one-half models with the appropriate symmetry boundary condition. The full spherical shell was reduced to a small section through the use of a spherical coordinate system and boundary conditions at the edge which permitted radial expansion but fixed motion in the theta and phi dimensions. The FEA models are shown in Figure 7. 

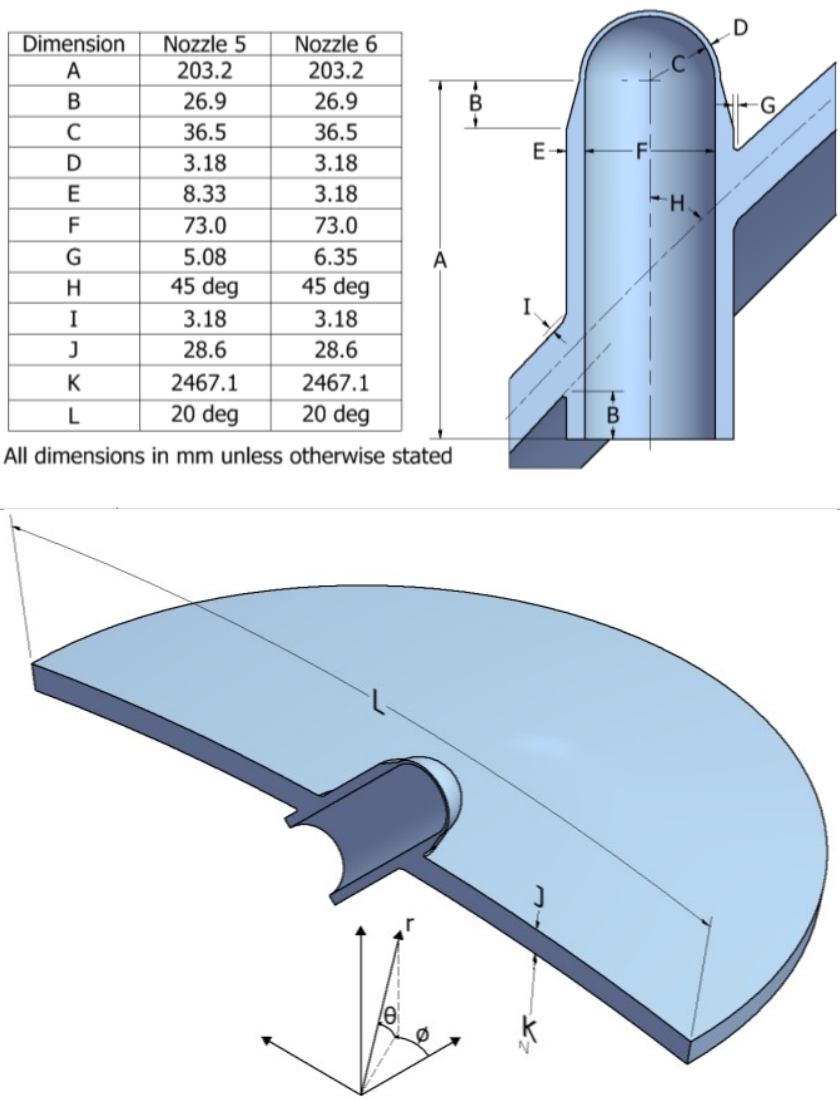

Figure 6 - Nozzle-Sphere Intersection Schematic and Dimensions

A perfectly plastic material model was adopted for the analysis using the yield stresses given in Table 3 . The ultimate tensile strength quoted shows that the material work hardens, but the absence of any further data prevents the use of hardening material models. Welded regions and heat affected zones very often have a higher yield stress than the surrounding parent material, but in this situation no information regarding this was provided. Therefore the material properties of the weld
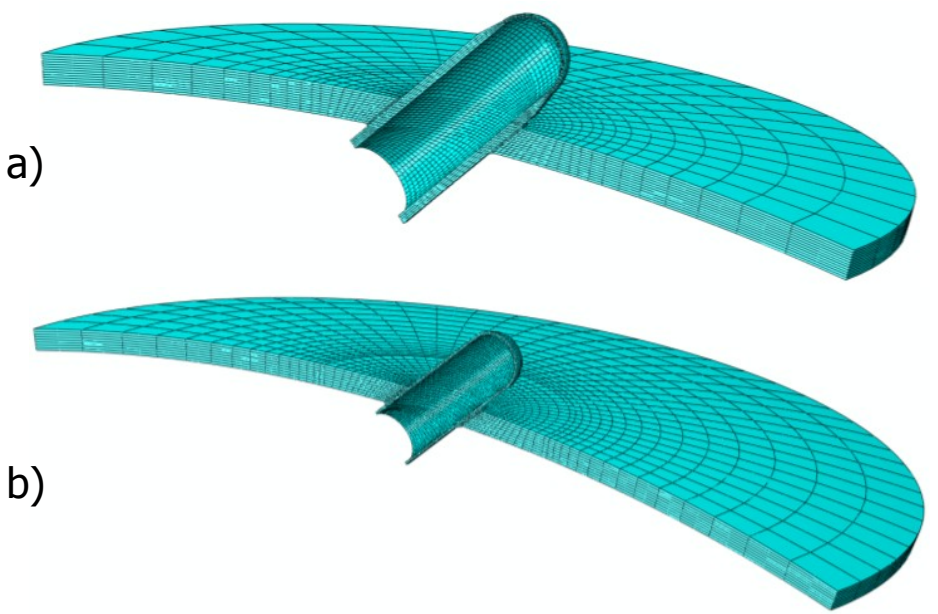

Figure 7 - Nozzle-Sphere Intersection FEA Models of a) Nozzle 5 and b) Nozzle 6

were assumed to be the same as those for the nozzle material which, being the lower of the two yield stresses, introduces a small conservatism into the analysis. 
An internal pressure was applied to all inner surfaces of the model. This pressure was established within a load cycle in the LMM analysis so that it would cycle from zero to a maximum pressure and then to zero once again. This load cycle is scaled by the LMM to find the shakedown limit, which in turn results in the shakedown pressure for the nozzle.

\subsection{Results Comparison}

Table 4 shows a comparison of the shakedown limit pressures found by experiment and through LMM calculation. The experimental lower bound corresponds to the highest level of cyclic pressure where shakedown was achieved. The experimental upper bound corresponds to the first cyclic pressure level where shakedown was not achieved.

Table 4 - Shakedown Pressure Comparison

\begin{tabular}{|c|c|c|c|c|}
\hline & \multicolumn{2}{|c|}{ LMM } & \multicolumn{2}{c|}{ Experiment } \\
\hline Nozzle & $\begin{array}{c}\text { Lower } \\
\text { Bound }\end{array}$ & $\begin{array}{c}\text { Upper } \\
\text { Bound }\end{array}$ & $\begin{array}{c}\text { Lower } \\
\text { Bound }\end{array}$ & $\begin{array}{c}\text { Upper } \\
\text { Bound }\end{array}$ \\
\hline 5 & 4.53 & 4.58 & 4.82 & 5.17 \\
\hline 6 & 4.12 & 4.16 & 4.48 & 4.82 \\
\hline
\end{tabular}

The shakedown pressures predicted by the LMM show reasonable agreement with the experiments whilst retaining a level of conservatism.

Figure 8 shows the location of the plastic strains in Nozzle 5, which are located at the nozzle-shell join, highlighting the reverse plasticity mechanism which would be observed when the cyclic pressure exceeds the shakedown pressure. This location was also highlighted in the C.E.G.B. report, which provides further verification of the LMM analysis. A similar correlation was also observed with Nozzle 6.

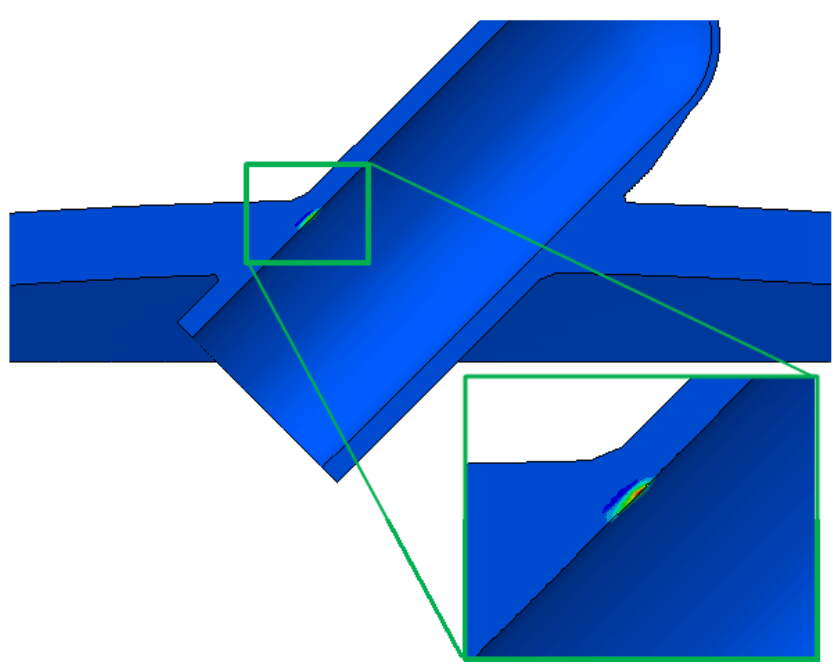

Figure 8 - Location of Reverse Plasticity in Nozzle 5

The conservatism seen in the predicted shakedown pressures is most likely to be due to the material model adopted in the LMM assessments. The perfectly plastic material does not capture any of the work hardening characteristics which may occur when the material is loaded beyond the elastic limit. The ultimate tensile strength of both materials shown in Table 3 is significantly higher than the yield stress, indicating that both materials show significant work hardening. In addition, with the repeated cycles of pressure, the material may also exhibit cyclic hardening which deviates further from the perfectly plastic assumption. Despite the very conservative material model the results obtained are still within $11 \%$, giving not only a conservative shakedown pressure but also the critical location in the structure in terms of plastic strains. 


\section{CONCLUSIONS AND FUTURE WORK}

This paper has presented comparisons of the LMM with experimental tests. Limit loads of pipe intersections subject to internal pressure and in-plane moment were considered first and two conclusions can be drawn from the results. Firstly, comparison of LMM and Abaqus limit analysis confirms the correct implementation of the bounding theorems. The second conclusion is that the LMM is capable of giving suitably conservative predictions of the limit load, which are effectively identical to those predicted by conventional non-linear FEA methods with the same modeling approximations. The ability to predict suitably conservative solutions was also found when the LMM was used beyond the capabilities of conventional FEA to predict the shakedown limit. The shakedown pressures of oblique nozzles in spherical shells were predicted with a reasonable yet conservative accuracy, despite the use of simplified material models. Furthermore, the location of the reverse plasticity mechanism predicted by the LMM correlated to that in the experiment, giving further confidence in the result.

The verifications presented here were deemed to be successful in verifying the LMM shakedown method as a tool for use in industry. In the immediate future it is important to consider more examples so that the method is verified using wide range of geometries and load histories. One specific example of this would be a thermally loaded example so that the temperature dependent material properties function of the LMM is used.

In addition to the shakedown analysis method, the LMM framework contains calculations for the ratchet limit for components operating beyond shakedown (i.e. where a reverse plasticity mechanism is involved) and for calculation of the stabilised response of the structure where the cycle contains a creep dwell. These methods contribute towards making the LMM a complete structural integrity tool for components operating at high temperatures. If this tool is to be adopted by industry in the future then the ratchet limit and creep calculation methods will also require validation with experimental results.

\section{ACKNOWLEDGMENTS}

The authors would like to thank the Engineering and Physical Sciences Research Council (EP/G038880/1), EDF Energy and the University of Strathclyde for their support in this research.

\section{REFERENCES}

1. R5: An assessment procedure for the high temperature response of structures, Revision 3, 2003, British Energy Generation Limited, Gloucester, UK.

2. Koiter, W.T., 1960. "General Theorems for Elastic Plastic Solids". In: Progress in Solid Mechanics, J.N. Sneddon, R. Hill, (Eds.), North Holland, Amsterdam, pp. 167-221.

3. Melan, E., 1936. "Theorie Statisch Unbestimmter Systeme aus Ideal-plastichem Baustoff". Sitzungsberichte der Kaiserliche Akademie der Wissenschaften in Wien 2A (145), 195-218.

4. Chen, H., 2010. "Lower and Upper Bound Shakedown Analysis of Structures with Temperature Dependent Material Properties". ASME Journal of Pressure Vessel Technology, 132(1), article 011202 (8 pages).

5. Chen, H., Ponter, A. R. S., 2010. "A Direct Method on the Evaluation of Ratchet Limit". ASME Joutnal of Pressure Vessel Technology, 132(4), article 041202 (8 pages).

6. Chen, H. Ure, J., Tipping, D., 2013. "Calculation of a Lower Bound Ratchet Limit Part 1 - Theory, Numerical Implementation and Verification". European Journal of Mechanics - A/Solids. 37, pp.361368.

7. Ure, J., Chen, H., Tipping, D., 2013. "Calculation of a Lower Bound Ratchet Limit Part 2 - Application to a Pipe Intersection with Dissimilar Material Join". European Journal of Mechanics - A/Solids. 37, pp.369-378.

8. Ellyin, F., 1976. "Experimental Investigation of Limit Loads of Nozzles in Cylindrical Vessels". Welding Research Council Bulletin, 219, pp.1-14.

9. Proctor, E., Flinders, F., 1968. "Shakedown Investigations on Partial Penetration Welded Nozzles in a Spherical Shell". Nuclear Engineering and Design, 8, pp.171-185.

10. Abaqus, 2009. "Users Manual". Dassault Systèmes Simulia Corp. 